\title{
Frequency Coupling and Sensitivity Spectral Measures of the Respiratory Sinus Arrhythmia System in Response to Increasing Respiratory Frequency
}

\author{
Alejandra Guillén-Mandujano ${ }^{1}$, Salvador Carrasco-Sosa ${ }^{1}$, Paola Coello-Caballero ${ }^{2}$ \\ ${ }^{1}$ Universidad Autónoma Metropolitana-I, CDMX, México, ${ }^{2}$ Universidad Politécnica de Chiapas, \\ Chiapas, México
}

\begin{abstract}
In 19 healthy subjects we assessed the effects of chirped respiratory frequency $(R F)$ from 0.05 to $0.8 \mathrm{~Hz}$, and of standing (STC) on the 130-s time courses of the central frequency and power of the high frequency components of $R R\left({ }_{C F E} H F_{R R}, P E H F_{R R}\right)$ and of respiration $\left({ }_{C F E} H F_{R E S},{ }_{P E} H F_{R E S}\right)$, estimated by a time-frequency distribution. We took as indexes of respiratory sinus arrhythmia (RSA) frequency coupling $\left(R S A_{F C}\right)$ the ${ }_{C F E} H F_{R E S^{-} C F E} H F_{R R}$ relation, their difference $\left(\triangle_{C F E} H F\right)$ and coherence $\left(R S A_{C O}\right)$, and the alpha index as RSA sensitivity $\left(R S A_{S}\right)$. The effects of RF on RSA measures were distinctive in three $R F$ ranges, with precise limits at $0.09 \pm 0.005,0.18 \pm 0.03,0.51 \pm 0.10$ and $0.81 \pm 0.03 \mathrm{~Hz}$. In the low, mid and high $R F$ ranges, respectively: ${ }_{C F E} H F_{R R}$ was first unchanged, proportional to $R F(r=0.97 \pm 0.03)$, then constant again; $R S A_{C O}$ was $0.73 \pm 0.06,0.97 \pm 0.03$ and $0.78 \pm 0.08 ; R S A_{S}$ was $135 \pm 34 \mathrm{~ms} / \mathrm{l}$, proportional to $R F(r=-0.79 \pm 0.08)$, and $62 \pm 30 \mathrm{~ms} / \mathrm{l} ; \Delta_{C F E} H F$ was greater than $0.02 \mathrm{~Hz}$ in the three RF stages. STC decreased mean $R S A_{S} \quad(p<0.02)$ in all $R F$ stages. $R S A_{F C}$ and $R S A_{S}$ measures vary as function of $R F$, showing three stages with precise $R F$ limits and distinctive functionality, respectively: low for $R S A_{F C}$ but high for $R S A_{S}$, optimal and linear for both, and reduced for both measures. Baroreflex activation significantly depresses $R S A_{S}$.
\end{abstract}

\section{Introduction}

The assessment of respiratory sinus arrhythmia (RSA) is widely used in clinical settings, spanning multiple diseases by system, by gender, or by development stage; however, the understanding of RSA physiology is still incomplete $[1,2]$. The physiological study of RSA has been usually performed by assessing the effects produced by changes in the respiratory variables, respiratory frequency $(\mathrm{RF})$ and tidal volume $\left(\mathrm{V}_{\mathrm{T}}\right)$, on the time and frequency domain measures of HRV. The studies on the effects of RF are more numerous and relevant than those of $\mathrm{V}_{\mathrm{T}}$. The most commonly used RSA measure is the power of the high frequency component of $\mathrm{HRV}\left({ }_{\mathrm{P}} \mathrm{HF}_{\mathrm{RR}}\right)$, considered a solid cardiac vagal activity marker [1].

Depending on the selected respiratory stimulation protocol and the spectral analysis methodology employed, the effect of different $\mathrm{RF}$ on ${ }_{\mathrm{P}} \mathrm{HF}_{\mathrm{RR}}$ has been characterized in continuous [3, 4] or discontinuous [5] manner. For the continuous approach, the breathing protocols that have been used did not control the $\mathrm{V}_{\mathrm{T}}$, and the RF changes were either executed in a pseudo-random fashion across the $0.05-0.5 \mathrm{~Hz}$ range [3] or decreasing RF from 0.46 to $0.05 \mathrm{~Hz}$, obtaining ${ }_{\mathrm{P}} \mathrm{HF}_{\mathrm{RR}}$ with a time-frequency distribution [4]. Both approaches have consistently characterized the effects of RF on ${ }_{\mathrm{P}} \mathrm{HF}_{\mathrm{RR}}$ as a nonlinear inverse relationship $[3,4,5]$.

We hypothesized that RSA function arises from the interaction of two mechanisms: a dynamic frequency coupling $\left(\mathrm{RSA}_{\mathrm{FC}}\right)$ that allows the central frequency of high frequency component of $\mathrm{HRV}\left({ }_{\mathrm{CF}} \mathrm{HF}_{\mathrm{RR}}\right)$ to follow the RF changes, and a gain mechanism ( $\left.\mathrm{RSA}_{S}\right)$, whose dynamic level determines, at a constant respiratory input, the amplitude of ${ }_{\mathrm{P}} \mathrm{HF}_{\mathrm{RR}}$ changes; and that the function of both mechanisms may be affected by the baroreflex mechanism (BR). To clarify the function and interaction of the two RSA mechanisms, we propose to assess them via novel spectral measures of RSAFC and $\mathrm{RSA}_{S}$, and to test their performance by analyzing their continuous response to chirped RF from 0.05 to $0.8 \mathrm{~Hz}$ at constant $\mathrm{V}_{\mathrm{T}}$, executed by the subjects in sitting (SIC) and standing (STC) conditions. Thus, we assessed the effects of the aforementioned maneuvers on the 130-s time courses of ${ }_{C F} \mathrm{HF}_{\mathrm{RR}}, \mathrm{PHF}_{\mathrm{RR}}$, the central frequency and power of the high frequency component of respiration $\left({ }_{\mathrm{CF}} \mathrm{HF}_{\mathrm{RES}}\right.$, ${ }_{\mathrm{P}} \mathrm{HF}_{\mathrm{RES}}$ ), estimated by a time-frequency distribution, from which the new RSA measures were computed.

\section{Methods}

\subsection{Subjects}

Nineteen healthy, normotensive and sedentary 
subjects, 11 men and 8 women, were studied. Mean age, height and weight were $22.2 \pm 2.2$ years, $167 \pm 8 \mathrm{~cm}$ and $69.1 \pm 10.4 \mathrm{~kg}$ respectively. Their written informed consent was requested to participate.

\subsection{Protocol}

Volunteers visited the laboratory twice. The first time, their health status and anthropometric variables were evaluated, and in the second visit the experimental stage was carried out. Volunteers underwent 20-s control, followed by a 90-s continuous and linear RF increase from 0.05 to $0.8 \mathrm{~Hz}$ at target $\mathrm{V}_{\mathrm{T}}$ of 0.61 for women and 0.81 for men; and a 20-s free-breathing recovery stage. The maneuver performance was visually guided by the chirp pattern displayed by an electronic device developed by us, and repeated in SIC and STC. ECG and $\mathrm{V}_{\mathrm{T}}$ were recorded throughout the protocol.

\subsection{Signal recording and acquisition}

ECG was detected at the CM5 bipolar lead using a bioelectric amplifier (Biopac). Respiration signal (Res) was obtained by integrating the flow signal (Validyne) provided by a pneumotachometer (Hans Rudolph). All signals were digitized at a sampling frequency of $1 \mathrm{kHz}$ via an acquisition and display system (Biopac).

\subsection{Data processing}

$\mathrm{R}$-wave peaks were detected to construct $\mathrm{R}-\mathrm{R}$ interval series (RR). Maxima and minima of each Res cycle were detected to form the $\mathrm{V}_{\mathrm{T}}$ and $\mathrm{RF}$ time series. RR, $\mathrm{V}_{\mathrm{T}}, \mathrm{RF}$ and Res were cubic-spline interpolated, resampled at $8 \mathrm{~Hz}$ and detrended. Time-frequency spectra of RR and Res were estimated with the smoothed pseudo-Wigner-Ville distribution. We extracted the instantaneous power and frequency of $\mathrm{RR}$ intervals and $\mathrm{V}_{\mathrm{T}}$ time series from the first two-order moments of their time-frequency distributions. To properly assess the response in the whole range of the RF sweep, $0.05-0.8 \mathrm{~Hz}$, we extended the HF band to that same range, thus obtaining the extended power and central frequencies of the highfrequency components of $\mathrm{RR}\left({ }_{\mathrm{PE}} \mathrm{HF}_{\mathrm{RR}}, \mathrm{CFE}_{\mathrm{CF}} \mathrm{HF}_{\mathrm{RR}}\right)$ and $\mathrm{Res}$ $\left({ }_{\mathrm{PE}} \mathrm{HF}_{\mathrm{Res}}, \mathrm{CFE}_{\mathrm{Hes}}\right)$. We computed the $\mathrm{CFE}_{\mathrm{CFE}} \mathrm{HF}_{\mathrm{RES}^{-} \mathrm{CFE}} \mathrm{HF}_{\mathrm{RR}}$ relation, the time courses of the difference between ${ }_{\mathrm{CFE}} \mathrm{HF}_{\mathrm{RES}}$ and ${ }_{\mathrm{CFE}} \mathrm{HF}_{\mathrm{RR}}\left(\Delta_{\mathrm{CFE}} \mathrm{HF}\right)$ and the ${ }_{\mathrm{PE}} \mathrm{HF}_{\mathrm{RR}-\mathrm{PE}} \mathrm{HF}_{\mathrm{RES}}$ coherence $\left(\mathrm{RSA}_{\mathrm{CO}}\right)$ as $\mathrm{RSA}_{\mathrm{FC}}$ indexes, and the alpha index (square root of the ${ }_{\mathrm{PE}} \mathrm{HF}_{\mathrm{RR}} /{ }_{\mathrm{PE}} \mathrm{HF}_{\mathrm{Res}}$ ratio) as $\mathrm{RSA}_{\mathrm{S}}$ measure.

The ensemble averages of the indexes dynamics clearly depicted a response pattern with three distinctive stages with sudden changes between each one, which allowed the detection of the RF limits that marked the stage transitions on each individual recording.

\subsection{Statistical analysis}

Data are expressed as mean \pm SD. Mean values of control, each stage of the response to the maneuver and recovery were computed. The indexes dynamics were used to compute linear regressions and correlations stage by stage and subject by subject, in SIC and STC. SIC vs. STC mean values differences were tested by paired t-test. Statistical significance was accepted at $\mathrm{p}<0.05$.

\section{Results}

${ }_{\mathrm{CFE}} \mathrm{HF}_{\mathrm{RES}}$, a spectral measure of Res series, showed a correlation of $0.992 \pm 0.007$ with RF, indicating that they are surrogate variables in different domains (Fig. 1).

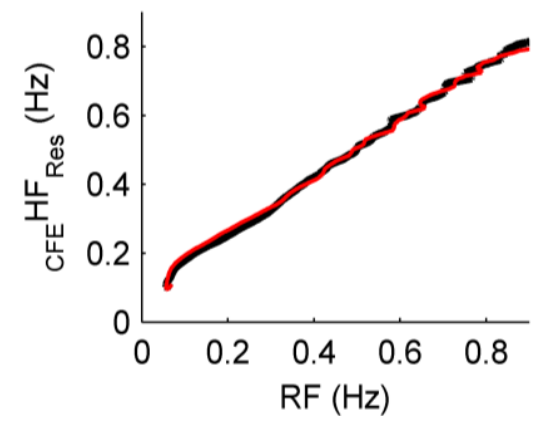

Fig.1. Ensemble averages of the relation between RF and ${ }_{\mathrm{CFE}} \mathrm{HF}_{\text {res }}$ in SIC (black) and STC (red).

The response of RSA spectral measures to chirped RF (Fig. 2A) in SIC presented three stages with sudden changes between them, with distinctive RF ranges -low, mid and high-, and effects. The mean values of the four RF limits detected were: $0.09 \pm 0.005 \mathrm{~Hz}, 0.18 \pm 0.03 \mathrm{~Hz}$, $0.51 \pm 0.10 \mathrm{~Hz}$ and $0.81 \pm 0.03 \mathrm{~Hz}$. Their characteristic effects on indexes with respect to control were: in the low $\mathrm{RF}$ range, $\mathrm{CFE}_{\mathrm{HF}} \mathrm{HF}_{\mathrm{RR}}$ was unchanged (Fig. $2 \mathrm{~B}$ ), $\Delta_{\mathrm{CFE}} \mathrm{HF}=-$ $0.10 \pm 0.04 \mathrm{~Hz}$ (Fig. 2C), RSA $\mathrm{CO}=0.73 \pm 0.06$ (Fig. 2D) and $\mathrm{RSA}_{\mathrm{S}} \quad(135 \pm 34 \mathrm{~ms} / \mathrm{l}$, Fig. 2E) showed a marked increment, achieving a maximal value; in the mid $\mathrm{RF}$ stage, $\mathrm{CFE}_{\mathrm{HR}}, \Delta_{\mathrm{CFE}} \mathrm{HF}(0.02 \pm 0.04 \mathrm{~Hz})$ and $\mathrm{RSA}_{\mathrm{S}}$ changed proportionally to RF (Fig. $2 \mathrm{~B}, \mathrm{C}$ and E), with a $\mathrm{RSA}_{\mathrm{CO}}$ of $0.97 \pm 0.03$ (Fig. 2D); in the high RF stage, ${ }_{\mathrm{CFE}} \mathrm{HF}_{\mathrm{RR}}$ remained elevated but constant (Fig. 2B), with a large $\Delta_{\mathrm{CFE}} \mathrm{HF}=0.33 \pm 0.6 \mathrm{~Hz}$ and depression in both $\mathrm{RSA} \mathrm{CO}_{\mathrm{CO}}$ (0.78 \pm 0.08 , Fig. 2D) and $\mathrm{RSA}_{\mathrm{S}}(62 \pm 30 \mathrm{~ms} / \mathrm{l}$, Fig.3E). In the three stages, ${ }_{P E} \mathrm{HF}_{\mathrm{RR}}$ (Fig. $2 \mathrm{~F}$ ) changed similarly to $\mathrm{RSA}_{S}$, due to ${ }_{\mathrm{PE}} \mathrm{HF}_{\mathrm{Res}}$ being constant (Fig. 2G). In the recovery, while ${ }_{\mathrm{CFE}} \mathrm{HF}_{\mathrm{RR}}(0.25 \pm 0.06 \mathrm{~Hz}$, Fig. $2 \mathrm{~B}), \Delta_{\mathrm{CFE}} \mathrm{HF}$ (0.16 \pm 0.09 Hz, Fig. 2C), RSA $\mathrm{CO}(0.67 \pm 0.08$, Fig. 2D) and ${ }_{\mathrm{PE}} \mathrm{HF}_{\mathrm{RR}}\left(8.0 \pm 1.2 \mathrm{~ms}^{2}\right.$, Fig. $\left.2 \mathrm{~F}\right)$ showed a gradual return to their baseline mean values, ${ }_{\mathrm{PE}} \mathrm{HF}_{\mathrm{Res}}\left(0.99 \pm 0.4 \mathrm{l}^{2}\right.$, Fig.3G) abruptly surpassed its baseline value and $\mathrm{RSA}_{\mathrm{S}}$ remained depressed (83 449 ms/l, Fig. 2E). 


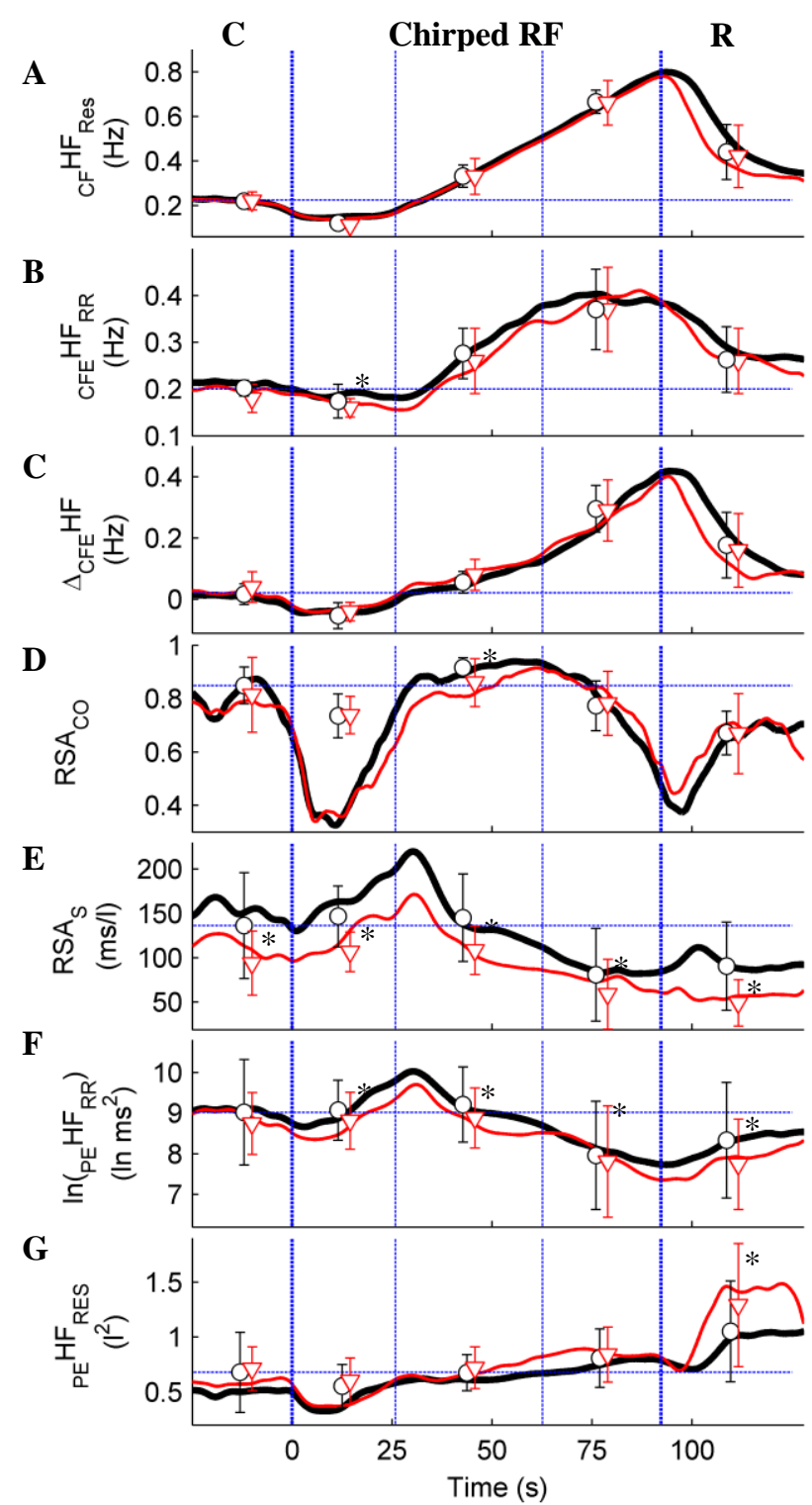

Fig. 2. Ensemble average and mean $\pm S D$ of the time courses of $\mathrm{RSA}_{\mathrm{FC}}$ and $\mathrm{RSA}_{\mathrm{S}}$ spectral measures in SIC (black) and STC (red). C=Control, R=Recovery. $* \mathrm{p}<0.025$ SIC vs. STC

Table 1 shows the mean values of the RF-RSA measures correlation coefficients and regression intercepts.

Table 1. Mean \pm SD correlation and regression intercept between RF and RSA spectral measures. $\mathrm{N}=19$.

\begin{tabular}{llll}
\hline Relation & Condition & $\mathrm{r}$ & Intercept \\
\hline${\text { RF- }{ }_{\mathrm{CFE}} \mathrm{HF}_{\mathrm{RR}}}$ & SIC & $0.97 \pm 0.03$ & $49 \pm 47$ \\
& STC & $0.93 \pm 0.05^{*}$ & $44 \pm 49^{*}$ \\
$\mathrm{RF}-\Delta_{\mathrm{CFE}} \mathrm{HF}$ & SIC & $0.86 \pm 0.10$ & $0.87 \pm 0.33$ \\
& STC & $0.62 \pm 0.40^{*}$ & $0.44 \pm 0.49^{*}$ \\
$\mathrm{RF}-\mathrm{RSA}$ & SIC & $-0.81 \pm 0.12$ & $245 \pm 115$ \\
& STC & $-0.79 \pm 0.24^{*}$ & $197 \pm 84^{*}$ \\
\hline
\end{tabular}

${ }^{*} \mathrm{p}<0.03$ SIC vs. STC
Chirped RF in STC, with respect to SIC, decreased $(\mathrm{p}<0.025)$ mean values of: ${ }_{\mathrm{CFE}} \mathrm{HF}_{\mathrm{RR}}$ in the low $\mathrm{RF}$ range (Fig. 2B), $\mathrm{RSA}_{\mathrm{CO}}$ in the mid $\mathrm{RF}$ range (Fig. 2D) and $\mathrm{RSA}_{S}$ in all of the RF ranges (Fig. 2E). In the mid RF stage, the correlations and intercepts of RF with ${ }_{\mathrm{CFE}} \mathrm{HF}_{\mathrm{RR}}$, $\Delta_{\mathrm{CFE}} \mathrm{HF}$ and $\mathrm{RSA}_{\mathrm{S}}$ regressions decreased (Table 1).

${ }_{C F E} \mathrm{HF}_{\mathrm{RES}^{-} \mathrm{CFE}} \mathrm{HF}_{\mathrm{RR}}$ relation clearly depicted the three stages that make up the patterned responses of RSA spectral measures to chirped RF. At RF below $0.09 \mathrm{~Hz}$ and above $0.5 \mathrm{~Hz}, \mathrm{CFE}_{\mathrm{CF}} \mathrm{HF}_{\mathrm{RR}}$ was constant. Only in the mid $\mathrm{RF}$ range, ${ }_{\mathrm{CFE}} \mathrm{HF}_{\mathrm{RR}}$ presented a correlation of $0.97 \pm 0.03$ with ${ }_{\mathrm{CFE}} \mathrm{HF}_{\mathrm{RES}}$. Because this relation (in both SIC and STC) was located below the identity line, for any given $\mathrm{RF}$ value the respective $\mathrm{CFE}_{\mathrm{CE}} \mathrm{HF}_{\mathrm{RR}}$ value was smaller. In SIC, the maximal ${ }_{\mathrm{CFE}} \mathrm{HF}_{\mathrm{RR}}$ was $0.46 \pm 0.07 \mathrm{~Hz}$. This relation was shifted downwards by STC (Fig. 3).

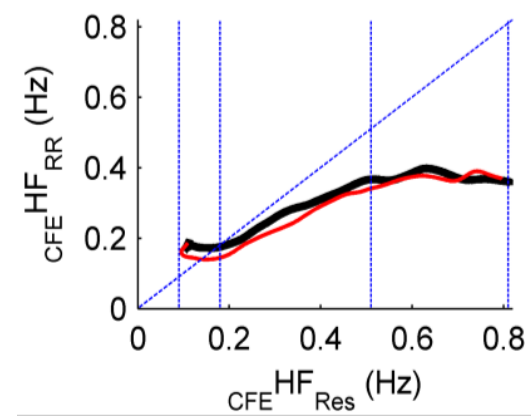

Fig. 3. Ensemble average of individual ${ }_{\mathrm{CFE}} \mathrm{HF}_{\mathrm{RES}}{ }^{-\mathrm{CFE}} \mathrm{HF}_{\mathrm{RR}}$ relations in SIC (black) and STC (red).

\section{Discussion}

The $\mathrm{RSA}_{\mathrm{FC}}$ and $\mathrm{RSA}_{\mathrm{S}}$ measures we used to characterize RSA mechanism show, in SIC and STC, a continuous patterned response to $0.05-0.8 \mathrm{~Hz}$ chirped RF that comprises three stages with distinctive functionality, at low, mid and high RF, limited at $0.9 \pm 0.005 \mathrm{~Hz}$, $0.18 \pm 0.03 \mathrm{~Hz}, \quad 0.51 \pm 0.10 \mathrm{~Hz}$ and $0.81 \pm 0.03 \mathrm{~Hz}$ respectively. In low RF stage, ${ }_{\mathrm{CFE}} \mathrm{HF}_{\mathrm{RR}}$ is unchanged and $\mathrm{RSA}_{S}$ shows an important increment; in mid RF stage ${ }_{C F E} \mathrm{HF}_{\mathrm{RR}}, \Delta_{\mathrm{CFE}} \mathrm{HF}$ and $\mathrm{RSA}_{\mathrm{S}}$ present strong correlations with RF. and in high RF stage ${ }_{C F E} \mathrm{HF}_{\mathrm{RR}}$ and $\mathrm{RSA}_{\mathrm{S}}$ stay constant. The ${ }_{\mathrm{CFE}} \mathrm{HF}_{\mathrm{Res}}{ }^{-\mathrm{CFE}} \mathrm{HF}_{\mathrm{RR}}$ relation shows clearly that in the three stages ${ }_{\mathrm{CFE}} \mathrm{HF}_{\mathrm{RR}}$ is smaller than ${ }_{\mathrm{CFE}} \mathrm{HF}_{\mathrm{Res}}$. Relative to SIC, increasing RF in STC reduced the mean values of $\mathrm{RSA}_{S}$ as well as the correlations and intercepts of $\mathrm{RF}$ with ${ }_{\mathrm{CFE}} \mathrm{HF}_{\mathrm{RR}}, \Delta_{\mathrm{CFE}} \mathrm{HF}$ and $\mathrm{RSA}_{\mathrm{S}}$ regressions.

In this study we extend the reported effects of a RF sweep from 0.05 to $0.5 \mathrm{~Hz}$ on ${ }_{\mathrm{P}} \mathrm{HF}_{\mathrm{RR}}[3,4]$ to the wider $0.05-0.8 \mathrm{~Hz}$ RF range, and to the novel $\mathrm{RSA}_{\mathrm{S}}$ and $\mathrm{RSA}_{\mathrm{FC}}$ indexes we propose. The patterned responses of these measures clearly depict three stages with distinct limits, each one characterized by presenting different degrees of gain and frequency coupling. Thus, the function of the RSA mechanism results from the dynamic interplay of $\mathrm{RSA}_{\mathrm{S}}$ and $\mathrm{RSA}_{\mathrm{FC}}$ mechanisms. 
After being obtained as gain by the transfer function method several years ago [3], RSA has rarely been used to evaluate Res effects on RSA. We have previously employed the alpha index method -commonly applied to calculate BR sensitivity- to compute $\mathrm{RSA}_{S}$ [6].

The function of $\mathrm{RSA}_{\mathrm{S}}$ and $\mathrm{RSA}_{\mathrm{FC}}$ mechanisms is optimal in the $0.18-0.51 \mathrm{~Hz}$ range, i.e., for each $\mathrm{RF}$ there is a proportional ${ }_{\mathrm{CFE}} \mathrm{HF}_{\mathrm{RR}}$ and $\mathrm{RSA}_{\mathrm{S}}$ value. In various disciplines, an usual method for studying RSA is obtaining ${ }_{\mathrm{P}} \mathrm{HF}_{\mathrm{RR}}$ during a paced breathing maneuver, with $\mathrm{RF}$ around $0.2 \mathrm{~Hz}$ and uncontrolled $\mathrm{V}_{\mathrm{T}}$, which yield results of dubious interpretability, because they only consider a single point of the wide and varying relationship between $\mathrm{RF}$ and ${ }_{\mathrm{P}} \mathrm{HF}_{\mathrm{RR}}$.

The reduced functionality of the $\mathrm{RSA}_{\mathrm{FC}}$ mechanism in the high $\mathrm{RF}$ range, for $\mathrm{RF}>0.64 \mathrm{~Hz}$ in SIC, may be partially attributed to the termed cardiac aliasing, which determines that RSA can only occur when the condition that $\mathrm{RF}<1 / 2$ mean heart rate (HR) is met [7], but not in STC, whose mean HR is greater.

In the low RF stage, there is a growing frequency coupling, associated to a large increase in the RSA gain mechanism due to the increment of ${ }_{\mathrm{PE}} \mathrm{HF}_{\mathrm{RR}}$, which reaches its maximal value. This effect has been named entrainment, due to the fusion of the low-frequency component of $\mathrm{HRV}$ with ${ }_{\mathrm{P}} \mathrm{HF}_{\mathrm{RR}}$ [8], a notion that fails to explain its significant reduction in STC. Thus, in this RF range, the function of the $\mathrm{RSA}_{\mathrm{FC}}$ mechanism is reduced, with a rapid increase toward the end of the stage. In the mid RF stage, our findings document that the frequency coupling and gain mechanisms change linearly with RF, although throughout the entire RF sweep ${ }_{\mathrm{CFE}} \mathrm{HF}_{\mathrm{RR}}$ is less than $\mathrm{RF}$, with a mean $\Delta_{\mathrm{CFE}} \mathrm{HF}$ of $0.02 \pm 0.04 \mathrm{~Hz}$. Therefore, in this stage, the functionality of the RSA mechanism is optimal, though towards the end of the stage it begins to decrease. In the high RF range the frequency coupling is reduced, ${ }_{C F E} \mathrm{HF}_{\mathrm{RR}}$ reaches a plateau of $0.46 \pm 0.06 \mathrm{~Hz}$ and $\mathrm{RSA}_{S}$ decreases to a minimum: RSA function is progressively depressed, even if $\mathrm{RF}<1 / 2 \mathrm{HR}$. At the end of the RF increase, and with its reflex reduction, the functionality of the RSA mechanisms is rapidly restored. This effect supports the reversibility of the RSA mechanisms. Due to the constancy of ${ }_{\mathrm{PE}} \mathrm{HF}_{\mathrm{Res}}$ across the three RF stages, $\mathrm{RSA}_{\mathrm{S}}$ and ${ }_{\mathrm{PE}} \mathrm{HF}_{\mathrm{RR}}$ changes are similar to one another, but in recovery, ${ }_{\mathrm{PE}} \mathrm{HF}_{\mathrm{Res}}$ quickly overtakes its baseline without provoking the expected marked increment of ${ }_{P E} \mathrm{HF}_{\mathrm{RR}}$, due to the depressed $\mathrm{RSA}_{S}$.

In STC, the limits of the stages are similar to those of SIC. RSA $\mathrm{FC}_{\mathrm{FC}}, \mathrm{RSA}_{\mathrm{S}}$ and their relations with $\mathrm{RF}$ are depressed, findings that evidentiate the close functional relationship between the RSA and BR mechanisms, both related to the vagal drive. BR activation by STC decreases vagal activity and, in consequence, decreases $\mathrm{RSA}_{\mathrm{S}}$ while altering $\mathrm{RSA}_{\mathrm{FC}}$. This strengthens the cardiac vagal withdrawal that, by accentuating the reduction of RSA, provokes the reduction of RR intervals and HRV, optimizing the cardiovascular adaptive response to STC.

The limits we found for the proportional part (mid RF range) add support to the correct selection of the frequency limits of the standard high frequency band.

In conclusion, in SIC and STC, RSA spectral measures vary as function of RF, showing three consecutive stages with characteristic RF limits and distinctive functionality, of some change, proportional change and no change. Specifically, the RSA mechanism: 1) In SIC shows a linear behavior in the $0.18-0.51 \mathrm{~Hz}$ range, notion supported by the strong correlations between $\mathrm{RSA}_{\mathrm{FC}}$ and $\mathrm{RSA}_{S}$ measures with RF; 2) presents a great increase in its gain at RF below $0.09 \mathrm{~Hz}$, and a large reduction above $0.51 \mathrm{~Hz} ; 3$ ) shows frequency uncoupling at low and high $\mathrm{RF}$ ranges, where ${ }_{\mathrm{CFE}} \mathrm{HF}_{\mathrm{RR}}$ is unchanged; and good frequency coupling in the medium RF range, even if at any given ${ }_{\mathrm{CFE}} \mathrm{HF}_{\mathrm{Res}}$, the ${ }_{\mathrm{CFE}} \mathrm{HF}_{\mathrm{RR}}$ is smaller; 4) is affected by the activation of the $\mathrm{BR}$ mechanism that depresses the $\mathrm{RSA}_{\mathrm{FC}}$ and $\mathrm{RSA}_{\mathrm{S}}$ measures, without altering the RF limits of the stages. Our findings support that the function of the RSA process results from the interaction and integration of its frequency coupling and gain mechanisms.

\section{References}

[1] Balzarotti S, Biassoni F, Colombo B, Ciceri M. Cardiac vagal control as a marker of emotion regulation in healthy adults: A review. Biol Psychol 2017; 130:54-66.

[2] Garcia A, Koschnitzky J, Dashevskiy T, Ramirez J. Cardiorespiratory coupling in health and disease. Auton Neurosci 2013; 175:26-37.

[3] Saul J, Berger R, Chen M, Cohen R. Transfer function analysis of autonomic regulation. II. Respiratory sinus arrhythmia. Am J Physiol 1989; 256:H153-H161.

[4] Novak V, Novak P, De Champlain J, Le Blanc A, et al. Influence of respiration on heart rate and blood pressure fluctuations. J Appl Physiol 1993; 74:617-626.

[5] Taylor J, Myers C, Halliwill J, Seidel H, Eckberg D. Sympathetic restraint of respiratory sinus arrhythmia: implications for vagal-cardiac tone assessment in humans. Am J Physiol 2001; 280:H2804-H2814.

[6] Carrasco-Sosa S, Guillén-Mandujano A, Rodríguez-Mejía A. Instantaneous response patterns of baroreflex sensitivity, respiratory sinus arrhythmia sensitivity and vagal activity to cold face test and active orthostatic test. CinC 2016; 43:111-114.

[7] Milde T, Schwab K, Walther M, Eiselt M, et al. Timevariant partial directed coherence in analysis of the cardiovascular system. A methodological study. Physiol Meas 2011; 32:1787-1805

[8] Pagani M, Lucini D, Rimoldi O, Furlan R, et al. Low and High Frequency Components of Blood Pressure Variability. Ann N Y Acad Sci 1996; 783:10-23.

Address for correspondence.

Alejandra Guillén-Mandujano

Depto. Ciencias de la Salud, T-172. UAM-I, CDMX, México.

ale.guillen.mandujano@gmail.com 